\title{
Semisynthetic modifications of antitubercular lanostane triterpenoids from Ganoderma
}

\author{
Panida Chinthanom ${ }^{1}$ - Vanicha Vichai ${ }^{1} \cdot K^{K a n c h a n a}$ Dokladda $^{1} \cdot$ Malipan Sappan ${ }^{1} \cdot$ Chawanee Thongpanchang $^{1}$. \\ Masahiko Isaka $\mathbb{( I D}^{1}$
}

Received: 19 January 2021 / Revised: 5 March 2021 / Accepted: 24 March 2021 / Published online: 12 May 2021

(c) The Author(s), under exclusive licence to the Japan Antibiotics Research Association 2021

\begin{abstract}
Antitubercular lanostane triterpenoids isolated from mycelial cultures of the basidiomycete Ganoderma australe were structurally modified by semisynthesis. One of the synthetic compounds, named GA003 (9), showed more potent activity against Mycobacterium tuberculosis H37Ra than the lead natural lanostane (1). GA003 was also significantly active against the virulent strain $(\mathrm{H} 37 \mathrm{Rv})$ as well as extensively drug-resistant tuberculosis strains.
\end{abstract}

\section{Introduction}

Tuberculosis (TB) remains one of the world's deadliest communicable diseases. In 2019, an estimated 10 million people fell in TB worldwide, and 1.4 million died from the disease [1]. Problematic issues in TB treatment include the increased incidence of multidrug-resistant TB (MDR-TB), which is a form of TB caused by bacteria that do not respond to isoniazid and rifampicin. Extensively drugresistant TB (XDR-TB) is a more serious form of MDR-TB caused by bacteria that do not respond to the most effective second-line anti-TB drugs, often leaving patients without any further treatment options. The increasing emergence of drug resistance highlights the need to develop novel chemical entity of TB drugs.

Ganoderma is a genus of bracket fungi including several medicinal mushroom species such as lingzhi (Ganoderma lucidum). Lanostane triterpenoids have been considered as one of the key ingredients of lingzhi, and a wide range of their biological activities related to human health promoting effects have been reported [2,3]. A number of lanostanes

Supplementary information The online version contains supplementary material available at https://doi.org/10.1038/s41429021-00422-5.

Masahiko Isaka

isaka@biotec.or.th

1 National Center for Genetic Engineering and Biotechnology (BIOTEC), Klong Luang, Pathumthani, Thailand and modified lanostanes have been isolated from natural or cultivated fruiting bodies and/or mycelial cultures of this genus $[3,4]$. We previously reported that several lanostanes from mycelial cultures of Ganoderma species exhibit significant growth inhibitory activities against Mycobacterium tuberculosis H37Ra [5-7]. In the conclusive full paper [5], the structure-activity relationships (SARs) of the natural lanostanes were proposed, wherein $(24 E)-3 \beta, 15 \alpha$-diacetoxylanosta-7,9(11),24-trien-26-oic acid (ganodermic acid $\mathrm{S}, \mathbf{1})$ was the optimal anti-TB Ganoderma lanostanoid (Fig. 1). In particular, $3 \beta$ - and $15 \alpha$-acetoxy groups were shown to be critical for the antimycobacterial activity. In a later study (unpublished), a methyl ester of $\mathbf{1}$ was inactive in the assay, which indicated the importance of the C-26 carboxyl group. On the basis of these results, we had planned semisynthetic modifications of compound $\mathbf{1}$ to discover more potent anti-TB agents.

\section{Results and discussion}

We had planned synthesis of derivatives of ganodermic acid $\mathrm{S}(\mathbf{1})$ [8] with various acyl groups at $\mathrm{C}-15$ and $\mathrm{C}-3\left(\mathrm{R}^{1}\right.$ and $\mathrm{R}^{2}$ in Fig. 1). As substrates for the semisynthesis, compounds $\mathbf{2}$ (ganodermic acid T-O) [9], $\mathbf{3}$ (ganodermic acid T$\mathrm{N}$ ) [9], and 4 (ganodermic acid $\mathrm{Jb}$ ) [10] were prepared (Fig. 2). In our previous chemical investigations of mycelial cultures of several Ganoderma species, Ganoderma australe BCC 22314 was selected as the most suitable sources of the desired substrates, since the strain produces $3 \beta, 15 \alpha-$

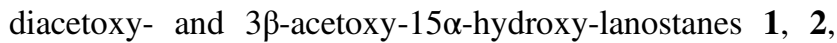


Fig. 1 Structure-activity relationships (SARs) for antitubercular activity of natural lanostanes from Ganoderma

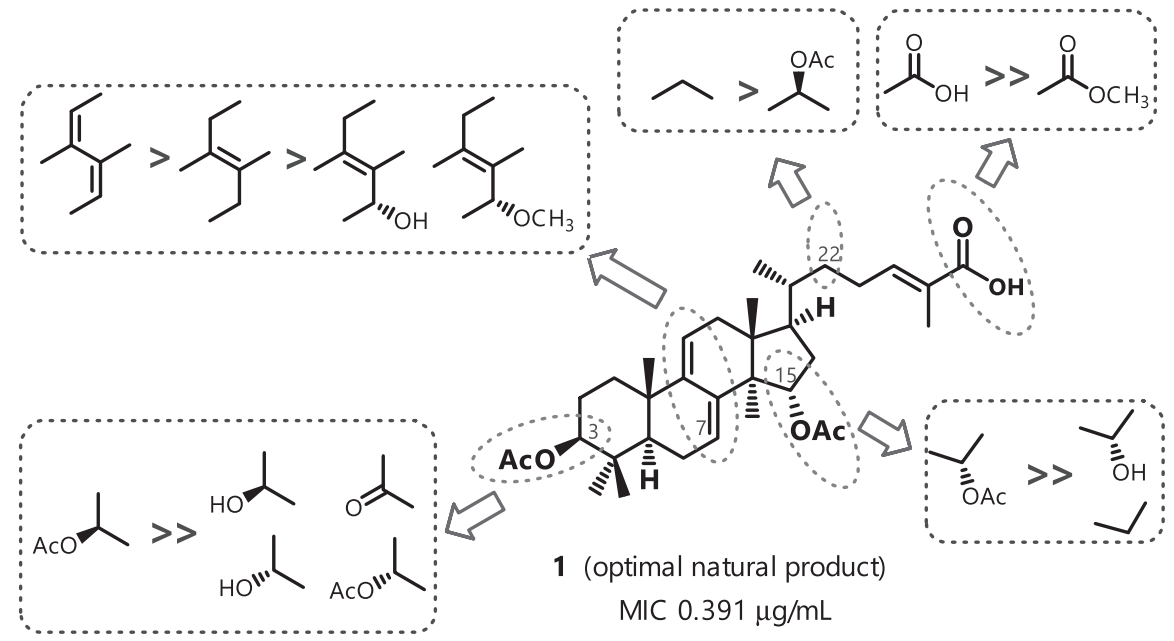

Semisynthetic Derivatization

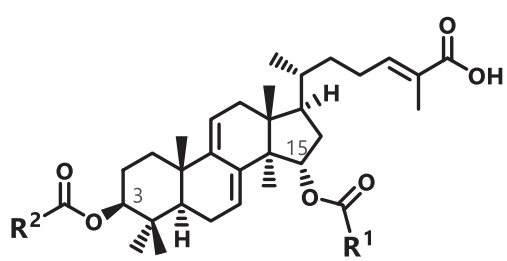

and 5-7 as major secondary metabolites. Compounds 5-7 are $7 \alpha$-hydroxy- 8 -ene or $7 \alpha$-acetoxy- 8 -ene derivatives that are acid sensitive and can be easily converted to 7,9(11)diene derivatives $\mathbf{1}$ and $\mathbf{2}$ under acidic conditions [7, 11]. For simplification of the lanostane compositions, the mycelial extract was treated with $p$-TsOH$\cdot \mathrm{H}_{2} \mathrm{O}$ in $\mathrm{CH}_{2} \mathrm{Cl}_{2}$ then the extract was subjected to chromatographic separations to furnish $\mathbf{1}$ and $\mathbf{2}$ as major lanostanes.

Compound 3 was prepared by partial alkaline hydrolysis of 1. Fortunately, 3-O-deacetylation was faster than 15-Odeacetylation; however, the regioselectivity of the hydrolysis was not so high. Therefore, the reaction was terminated at an appropriate stage to obtain a mixture mainly composed of $\mathbf{1}, \mathbf{3}$, and $\mathbf{4}$. Compound $\mathbf{4}$ was synthesized by complete hydrolysis of $\mathbf{1}$.

Derivatives 8-18 (Fig. 3) were synthesized using compounds 2-4. The formylated derivatives 8 and 13 were successfully prepared from $\mathbf{2}$ and $\mathbf{3}$, respectively, by using formic acid-2,2,2-trifluoroethyl formate [12]. All other acylated derivatives were synthesized by acylation of 2-4 with excess acyl chloride in pyridine- $\mathrm{CH}_{2} \mathrm{Cl}_{2}$.

Semisynthetic derivatives 8-18 were tested for activity against $M$. tuberculosis H37Ra (Table 1). Most notably, 15-Opropionyl derivative 9, named GA003, showed higher antimycobacterial activity (MIC $0.0977 \mu \mathrm{g} / \mathrm{ml}$ ) than 1 . Comparison of the activities of $\mathbf{1}$ and 8-12, possessing different 15-Oacyl $\left(\mathrm{R}^{1}\right)$, demonstrated that propionyl $\left(\mathrm{R}^{1}=\mathrm{Et}\right)$ derivative $(\mathbf{9})$ has the most suitable size of the $\mathrm{R}^{1}$ alkyl group. Similarly, the activities of $\mathbf{1}$ and 13-16 with different 3-O-acyl $\left(\mathrm{R}^{2}\right)$ suggested that acetyl $\left(\mathbf{1}, \mathrm{R}^{2}=\mathrm{Me}\right)$ and propionyl $\left(\mathbf{1 4}, \mathrm{R}^{2}=\mathrm{Et}\right)$ have suitable size of $\mathrm{R}^{2}$ alkyl group. The activities of dipropionyl and dibutyryl derivatives $\mathbf{1 7}$ and $\mathbf{1 8}$ were reasonably consistent with these partial SARs.

Cytotoxicity of these compounds to noncancerous Vero cells (African green monkey kidney fibroblasts) was also evaluated. Compound $\mathbf{9}$ and many other compounds showed moderate or weak cytotoxicity; however, there were reasonable selectivity with the antimycobacterial activities. The SAR of cytotoxicity to Vero cells was different from that for the anti-TB activity, which indicated that the positive assay results for growth inhibition of TB mycobacteria are not related to the cytotoxic properties of the lanostanes.

The most potent antimycobacterial compound 9 (GA003) also showed significant activity against the virulent strain M. tuberculosis H37Rv (ATCC 27294) with an MIC value of $0.313 \mu \mathrm{g} \mathrm{ml}{ }^{-1}$. Then, it was further tested for activities against drug-resistant strains of $M$. tuberculosis, pre-XDR strain (THX-0001), and XDR strains (THX-0002, THX0003). GA003 was active against these strains exhibiting MIC values of $0.313,0.625$, and $1.25 \mu \mathrm{g} \mathrm{ml}^{-1}$, respectively.

In the present study, derivatives of the natural anti-TB lanostane 1 were prepared by semisynthesis. Among them, GA003 (9) exhibited superior activity than $\mathbf{1}$ against $M$. tuberculosis H37Ra. This compound was active against the 
Fig. 2 Preparation of $\mathbf{2}, \mathbf{3}$, and $\mathbf{4}$ as substrates for semisynthesis of derivatives of $\mathbf{1}$. Reagents and conditions: (a) the mycelial extract was treated with $p$-TsOH. $\mathrm{H}_{2} \mathrm{O}$ in $\mathrm{CH}_{2} \mathrm{Cl}_{2}, \mathrm{rt}, 1 \mathrm{~h}$; (b) $2 \mathrm{M}$ $\mathrm{NaOH}-\mathrm{MeOH}$ (1:6), rt, 5 h; (c) $3 \mathrm{M} \mathrm{NaOH}-\mathrm{MeOH}(1: 10)$,

$\mathrm{rt}, 23 \mathrm{~h}$
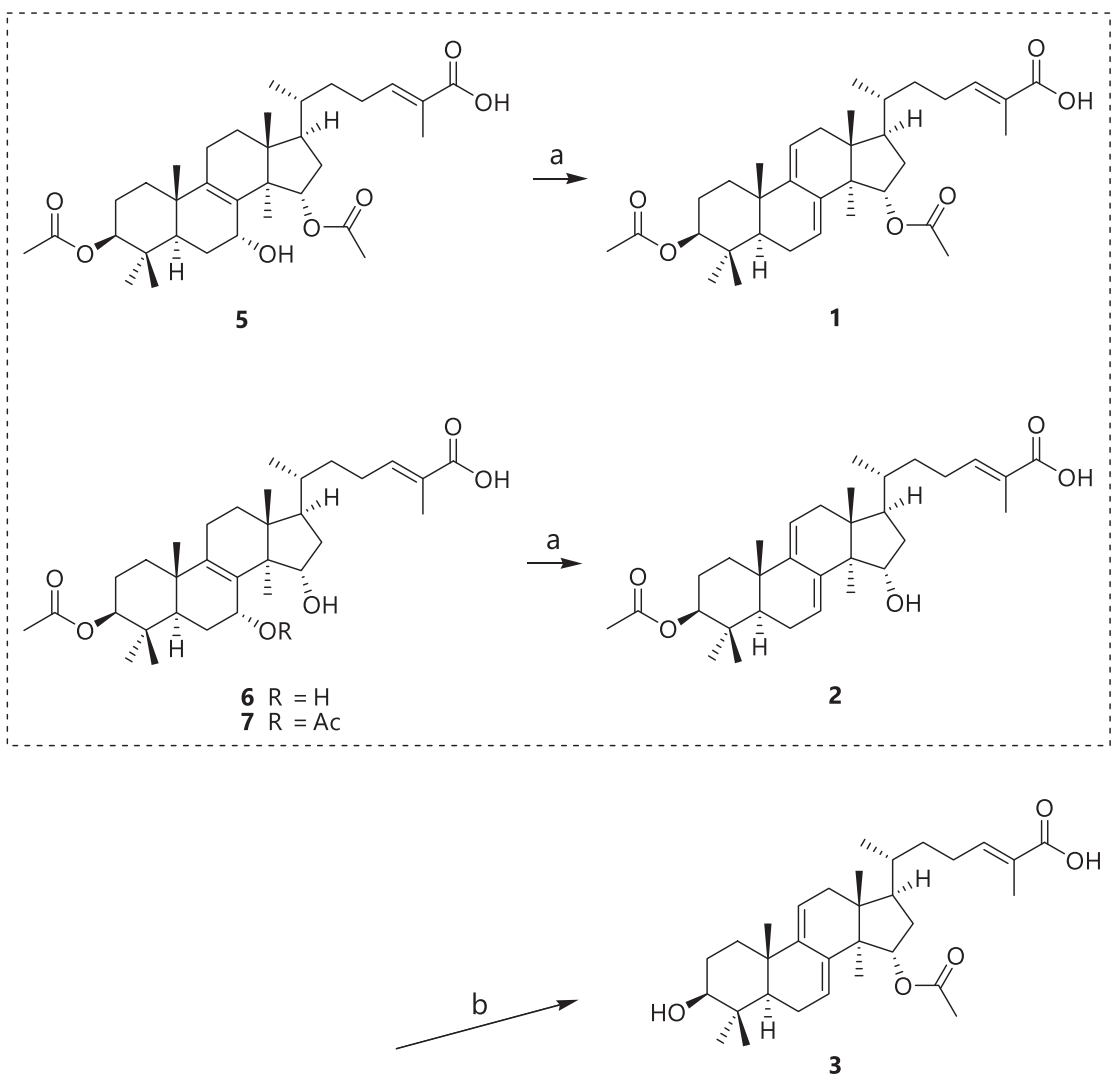

1

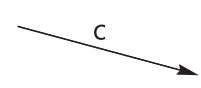<smiles>C/C(=C\CC[C@H](C)[C@H]1C[C@H](O)[C@]2(C)C3=CC[C@H]4C(C)(C)[C@@H](O)CC[C@]4(C)C3=CC[C@]12C)C(=O)O</smiles>

virulent strain M. tuberculosis $\mathrm{H} 37 \mathrm{Rv}$, moreover, also against the XDR strains. The MIC values against XDR strains were relatively larger than those against drugsensitive strains. However, considering the urgent need for the discovery of novel chemotypes against drug-resistant $\mathrm{TB}$, these activities will be reasonably good enough to consider further biochemical and pharmacological evaluations as a lead compound.

\section{Materials and methods}

\section{General experimental procedures}

Optical rotations were determined using a JASCO P-2000 digital polarimeter. FTIR spectra were acquired using a Bruker ALPHA spectrometer. NMR spectra were recorded on a Bruker DRX400 spectrometer. ESI-TOF mass spectra were measured using a Bruker micrOTOF mass spectrometer.

\section{Fungal material}

Ganoderma australe was isolated from a dead oil palm (Elaeis guineensis) trunk in the plantation area, Klong Thom, Krabi Province, Thailand, on May 4, 2006. The mushroom specimen was deposited in the BIOTEC Bangkok Herbarium as BBH 19074, and the living culture was deposited in the BIOTEC Culture Collection as BCC 22314. Identification of this fungus is based on the morphology and ITS rDNA sequence data (GenBank accession number: KX421866) [5].

\section{Fermentation of G. australe BCC 22314, extraction, and isolation of 1 and 2}

The fungus BCC 22314 was maintained on potato dextrose agar at $25^{\circ} \mathrm{C}$. The agar was cut into small plugs and inoculated into $12 \times 250$-ml Erlenmeyer flasks containing 25 $\mathrm{ml}$ of malt extract broth (MEB; malt extract $6.0 \mathrm{~g} \mathrm{l}^{-1}$, yeast 
Fig. 3 Synthesis of the derivatives of $\mathbf{1}$. Reagents and conditions: (a) formic acid-2,2,2-trifluoroethyl formate, $60{ }^{\circ} \mathrm{C}, 3 \mathrm{~h}$; (b) $\mathrm{RCOCl}$ (excess), pyridine- $\mathrm{CH}_{2} \mathrm{Cl}_{2}(2: 1)$, $\mathrm{rt}, 3 \mathrm{~h}$

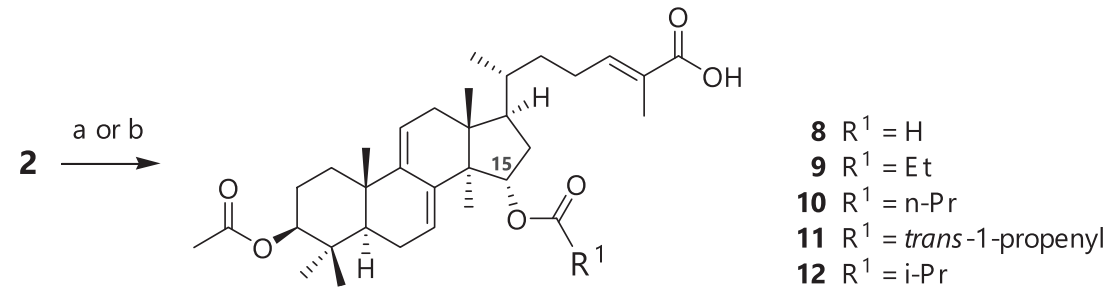

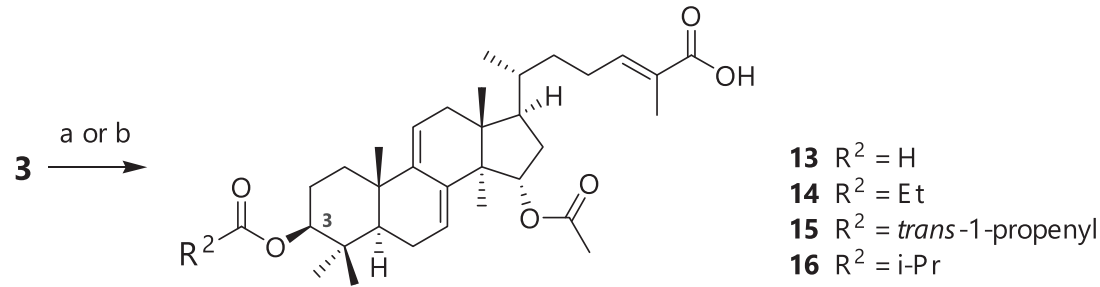

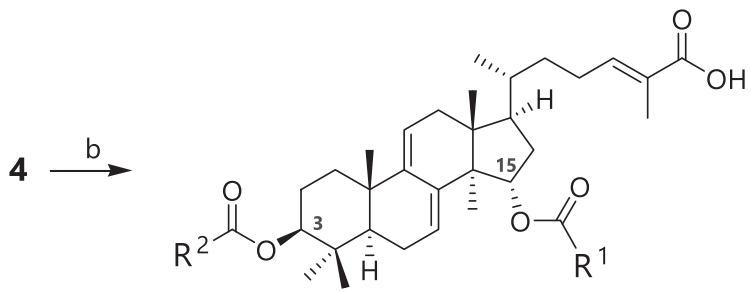
$18 R^{1}=R^{2}=n-P r$
Table 1 Antimycobacterial and cytotoxic activities of the lanostanes

\begin{tabular}{lll}
\hline & Antituberculosis & Cytotoxicity \\
\hline Compound & M. tuberculosis H37Ra H37Ra & Vero cells \\
& MIC, $\mu \mathrm{g} \mathrm{ml}^{-1}$ & $\mathrm{IC}_{50}, \mu \mathrm{g} \mathrm{ml}^{-1}$ \\
$\mathbf{1}$ & 0.391 & 18 \\
$\mathbf{8}$ & 3.13 & 15 \\
$\mathbf{9}$ (GA003) $^{-13}$ & 0.0977 & 9.2 \\
$\mathbf{1 0}$ & 1.56 & 12 \\
$\mathbf{1 1}$ & 0.781 & 12 \\
$\mathbf{1 2}$ & 0.781 & 9.5 \\
$\mathbf{1 3}$ & 1.56 & 16 \\
$\mathbf{1 4}$ & 0.391 & 16 \\
$\mathbf{1 5}$ & $>50$ & 18 \\
$\mathbf{1 6}$ & 0.781 & 15 \\
$\mathbf{1 7}$ & 0.781 & 8.7 \\
$\mathbf{1 8}$ & $>50$ & 13 \\
Rifampicin $^{\mathrm{a}}$ & 0.025 & - \\
Isoniazid $^{\mathrm{a}}$ & 0.0469 & - \\
Ellipticine $^{\mathrm{b}}$ & - & 1.3 \\
\hline
\end{tabular}

${ }^{\text {a Standard anti-TB drugs }}$

${ }^{\mathrm{b}}$ Standard compound for the cytotoxicity assay extract $1.2 \mathrm{~g}^{-1}$, maltose $1.8 \mathrm{~g}^{-1}$, dextrose $6.0 \mathrm{~g}^{-1}$ ). After incubation at $25^{\circ} \mathrm{C}$ for 7 days on a rotary shaker $(200 \mathrm{rpm}$ ), each primary culture was transferred into a 1000-ml Erlenmeyer flask containing $250 \mathrm{ml}$ of the same liquid medium (MEB), and incubated at $25^{\circ} \mathrm{C}$ for 7 days on a rotary shaker (200 rpm). The secondary cultures were pooled and each 25 ml portion was transferred into $120 \times 1000$-ml Erlenmeyer flasks containing $250 \mathrm{ml}$ of MEB, and the final fermentation was carried out at $25^{\circ} \mathrm{C}$ for 90 days under static conditions. The cultures were filtered, and the residual wet mycelia were macerated in acetone $\left(61,25^{\circ} \mathrm{C}, 7\right.$ days $)$ and filtered. The filtrate was concentrated under reduced pressure, and the residue was diluted with $\mathrm{H}_{2} \mathrm{O}(600 \mathrm{ml})$ and extracted with EtOAc (2.01). The EtOAc solution was concentrated under reduced pressure to obtain a brown gum (mycelial extract, $6.30 \mathrm{~g})$. This extract was dissolved in $\mathrm{CH}_{2} \mathrm{Cl}_{2}(50 \mathrm{ml})$ and $p$ $\mathrm{TsOH} \cdot \mathrm{H}_{2} \mathrm{O}(74 \mathrm{mg})$ was added. After stirring for $1 \mathrm{~h}, \mathrm{H}_{2} \mathrm{O}$ $(20 \mathrm{ml})$ and $28 \%$ aqueous ammonia solution $(0.2 \mathrm{ml})$ were added, and then partially concentrated by evaporation. The residual aqueous solution was diluted with EtOAc $(100 \mathrm{ml})$ and washed with $\mathrm{H}_{2} \mathrm{O}(50 \mathrm{ml})$. The $\mathrm{H}_{2} \mathrm{O}$ layer was extracted with EtOAc $(2 \times 100 \mathrm{ml})$. The combined organic layer was concentrated under reduced pressure to obtain a brown gum 
(acid-treated mycelial extract; $6.14 \mathrm{~g}$ ). This extract was subjected to column chromatography on silica gel (EtOAc-hexane, step gradient elution from 0:100 to 100:0), then the fractions containing the target compounds were further purified by preparative HPLC (Waters SunFire GromSil 120 ODS- 4 HE, $20 \times 150 \mathrm{~mm}, 5 \mu \mathrm{m}$; mobile phase $\mathrm{MeCN}-\mathrm{H}_{2} \mathrm{O}$, gradient from 70:30 to 100:0 over $30 \mathrm{~min}$; flow rate $\left.12 \mathrm{ml} \mathrm{min}^{-1}\right)$ to furnish $\mathbf{1}(792 \mathrm{mg})$ and $2(511 \mathrm{mg})$.

\section{Preparation of $\mathbf{3}$ by partial alkaline hydrolysis of 1}

Compound 1 (103 mg, $0.19 \mathrm{mmol}$ ) was partially hydrolyzed in $2 \mathrm{M} \mathrm{NaOH}(1 \mathrm{ml})-\mathrm{MeOH}(6 \mathrm{ml})$ at room temperature for $5 \mathrm{~h}$. The mixture was acidified to $\mathrm{pH} 2$ by addition of 1 $\mathrm{M} \mathrm{HCl}$ and partially concentrated by evaporation. The residue was diluted with $\mathrm{H}_{2} \mathrm{O}$ and extracted with EtOAc. The organic layer was concentrated under reduced pressure to obtain a pale yellow gum, which was subjected to column chromatography on silica gel (EtOAc-hexane) to furnish $\mathbf{3}$ (27 mg, 28\%).

\section{Preparation of 4 by complete alkaline hydrolysis of 1}

Compound 1 (56 mg, $0.10 \mathrm{mmol}$ ) was completely hydrolyzed in $3 \mathrm{M} \mathrm{NaOH}(0.4 \mathrm{ml})-\mathrm{MeOH}(4 \mathrm{ml})$ at room temperature for $23 \mathrm{~h}$. The mixture was acidified by addition of $1 \mathrm{M} \mathrm{HCl}$ and partially concentrated by evaporation. The residual aqueous solution was diluted with $\mathrm{H}_{2} \mathrm{O}$ and extracted with EtOAc. The organic layer was concentrated in vacuo to obtain 4 (46 mg, 97\%).

\section{Synthesis of 8}

A mixture of $2(8.6 \mathrm{mg}, 17 \mu \mathrm{mol})$ and formic acid $(20 \mu \mathrm{l})$ in 2,2,2-trifluoroethyl formate $(0.2 \mathrm{ml})$ was stirred on a $60^{\circ} \mathrm{C}$ heating bath for $3 \mathrm{~h}$. After cooling to room temperature, the mixture was concentrated in vacuo. The residue $(10 \mathrm{mg})$ was purified by preparative HPLC $\left(\mathrm{MeCN}-\mathrm{H}_{2} \mathrm{O}\right)$ to furnish 8 (3.0 mg, 33\%).

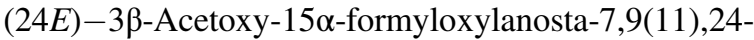
trien-26-oic acid (8): colorless amorphous solid; IR (ATR) $\nu_{\max } 2929,1724,1686,1375,1247,1177 \mathrm{~cm}^{-1} ;{ }^{1} \mathrm{H}$ NMR $\left(400 \mathrm{MHz}, \mathrm{CDCl}_{3}\right)$, selected resonances, $\delta 8.11(1 \mathrm{H}, \mathrm{s})$, $6.84(1 \mathrm{H}, \mathrm{t}, J=7.0 \mathrm{~Hz}), 5.50(1 \mathrm{H}, \mathrm{d}, J=6.0 \mathrm{~Hz}), 5.33(1 \mathrm{H}$, $\mathrm{d}, J=5.7 \mathrm{~Hz}), 5.19(1 \mathrm{H}, \mathrm{dd}, J=9.9,5.4 \mathrm{~Hz}), 4.51(1 \mathrm{H}, \mathrm{dd}$, $J=11.2,4.5 \mathrm{~Hz}), 2.33(1 \mathrm{H}$, br d, $J=16.7 \mathrm{~Hz}), 2.32(2 \mathrm{H}, \mathrm{t}$, $J=7.5 \mathrm{~Hz}), 2.06(3 \mathrm{H}, \mathrm{s}), 1.83(3 \mathrm{H}, \mathrm{s}), 1.17(1 \mathrm{H}, \mathrm{dd}, J=$ 11.3, 4.2 Hz), $1.03(3 \mathrm{H}, \mathrm{s}), 0.99(3 \mathrm{H}, \mathrm{s}), 0.94(3 \mathrm{H}, \mathrm{s}), 0.92$ $(3 \mathrm{H}, \mathrm{d}, J=6.4 \mathrm{~Hz}), 0.89(3 \mathrm{H}, \mathrm{s}), 0.66(3 \mathrm{H}, \mathrm{s}) ;{ }^{13} \mathrm{C} \mathrm{NMR}$ $\left(100 \mathrm{MHz}, \mathrm{CDCl}_{3}\right) \delta 172.1,170.9,161.1,145.8,144.8$, $140.2,126.9,121.3,116.2,80.7,77.2,51.6,49.2,49.0$, $44.2,38.1,37.7,37.4,37.0,36.0,35.5,34.7,28.1,25.9$, $24.3,22.9,22.8,21.2,18.4,18.2,16.9,16.0,12.1$; HRMS

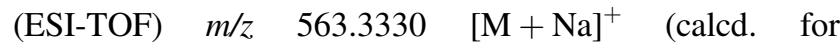
$\mathrm{C}_{33} \mathrm{H}_{48} \mathrm{O}_{6} \mathrm{Na}, 563.3343$ ).

\section{Synthesis of 9 (GA003)}

Compound 2 (64 mg, $0.125 \mathrm{mmol})$ was treated with propionyl chloride $(0.20 \mathrm{ml})$ in pyridine $(0.8 \mathrm{ml}) / \mathrm{CH}_{2} \mathrm{Cl}_{2}(0.80$ $\mathrm{ml}$ ) at room temperature for $3 \mathrm{~h}$. The mixture was diluted with EtOAc $(50 \mathrm{ml})$ and washed with $\mathrm{H}_{2} \mathrm{O}(3 \mathrm{ml})$. The organic layer was concentrated under reduced pressure to obtain a pale yellow solid, which was purified by preparative HPLC (Waters SunFire Prep C18 OBD, 19×250 $\mathrm{mm}, 10 \mu \mathrm{m}$; gradient of $\mathrm{MeCN}-\mathrm{H}_{2} \mathrm{O}$ from 80:20 to $100: 0$ over $30 \mathrm{~min}$; flow rate $12 \mathrm{ml} / \mathrm{min})$ to furnish $9(32 \mathrm{mg}, 45 \%$; $\left.t_{\mathrm{R}} 26 \mathrm{~min}\right)$.

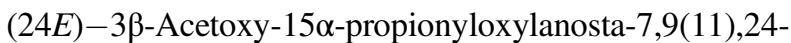
trien-26-oic acid (9, GA003): colorless solid; $[\alpha]^{22}{ }_{D}+99(c$ $0.17, \mathrm{CHCl}_{3}$ ); IR (ATR) $\nu_{\max } 2936,1731,1686,1375,1247$, $1029 \mathrm{~cm}^{-1} ;{ }^{1} \mathrm{H}$ NMR $\left(\mathrm{CDCl}_{3}, 400 \mathrm{MHz}\right) \delta 6.85(1 \mathrm{H}, \mathrm{t}, J=$ $7.0 \mathrm{~Hz}, \mathrm{H}-24), 5.46(1 \mathrm{H}, \mathrm{d}, J=4.8 \mathrm{~Hz}, \mathrm{H}-7), 5.31(1 \mathrm{H}, \mathrm{d}$, $J=5.9 \mathrm{~Hz}, \mathrm{H}-11), 5.07(1 \mathrm{H}, \mathrm{dd}, J=9.8,5.0 \mathrm{~Hz}, \mathrm{H}-15), 4.50$ $(1 \mathrm{H}, \mathrm{dd}, J=11.3,4.5 \mathrm{~Hz}, \mathrm{H}-3), 2.36(2 \mathrm{H}, \mathrm{q}, J=7.5 \mathrm{~Hz}$, propionyl $\left.\mathrm{H}-2^{\prime \prime}\right), 2.32\left(1 \mathrm{H}\right.$, br d, $\left.J=16.5 \mathrm{~Hz}, \mathrm{H}_{\alpha}-12\right), 2.23$ $\left(1 \mathrm{H}, \mathrm{m}, \mathrm{H}_{\mathrm{a}}-23\right), 2.11\left(1 \mathrm{H}, \mathrm{m}, \mathrm{H}_{\beta}-16\right), 2.10\left(1 \mathrm{H}, \mathrm{m}, \mathrm{H}_{\mathrm{b}}\right.$ -23), $2.08\left(1 \mathrm{H}, \mathrm{m}, \mathrm{H}_{\beta}-6\right), 2.06(3 \mathrm{H}, \mathrm{s}$, acetyl $\mathrm{H}-2), 2.05$ $\left(1 \mathrm{H}, \mathrm{m}, \mathrm{H}_{\alpha}-6\right), 1.99\left(1 \mathrm{H}, \mathrm{m}, \mathrm{H}_{\beta}-1\right), 1.83(3 \mathrm{H}, \mathrm{s}), 1.72(1 \mathrm{H}$, $\left.\mathrm{m}, \mathrm{H}_{\alpha}-2\right), 1.70-1.67\left(3 \mathrm{H}, \mathrm{m}, \mathrm{H}_{\beta}-2, \mathrm{H}_{\alpha}-16\right.$, and $\mathrm{H}-17$ ), 1.53-1.50 (2H, m, $\mathrm{H}_{\alpha}-1$ and $\left.\mathrm{H}_{\mathrm{a}}-22\right), 1.40(1 \mathrm{H}, \mathrm{m}, \mathrm{H}-20)$, $1.17(1 \mathrm{H}, \mathrm{m}, \mathrm{H}-5), 1.17\left(3 \mathrm{H}, \mathrm{t}, J=7.5 \mathrm{~Hz}\right.$, propionyl $\left.\mathrm{H}-3^{\prime}\right)$, $1.13\left(1 \mathrm{H}, \mathrm{m}, \mathrm{H}_{\mathrm{b}}-22\right), 1.00(3 \mathrm{H}, \mathrm{s}, \mathrm{H}-30), 0.98(3 \mathrm{H}, \mathrm{s}, \mathrm{H}-$ 19), 0.94 (3H, s, H-29), 0.91 (3H, d, $J=6.4 \mathrm{~Hz}, \mathrm{H}-21), 0.89$ (3H, s, H-28), 0.65 (3H, s, H-18); ${ }^{13} \mathrm{C}$ NMR $\left(\mathrm{CDCl}_{3}, 100\right.$ MHz) $\delta 174.5$ (C, propionyl C-1"), 172.5 (C, C-26), 171.0 (C, acetyl C-1'), 145.6 (C, C-9), 145.1 (CH, C-24), 140.2 (C, C-8), 126.7 (C, C-25), 121.1 (CH, C-7), 116.1 (CH, C-11), 80.7 (CH, C-3), 77.1 (CH, C-15), 51.4 (C, C-14), 48.9 (CH, C-17), 48.8 (CH, C-5), 44.1 (C, C-13), $37.9\left(\mathrm{CH}_{2}, \mathrm{C}-12\right)$, 37.6 (C, C-4), 37.3 (C, C-10), $37.1\left(\mathrm{CH}_{2}, \mathrm{C}-16\right), 35.9(\mathrm{CH}$, $\mathrm{C}-20), 35.3\left(\mathrm{CH}_{2}, \mathrm{C}-1\right), 34.6\left(\mathrm{CH}_{2}, \mathrm{C}-22\right), 28.1\left(\mathrm{CH}_{3}, \mathrm{C}-28\right)$, $28.0\left(\mathrm{CH}_{2}\right.$, propionyl C-2"), $25.9\left(\mathrm{CH}_{2}, \mathrm{C}-23\right), 24.2\left(\mathrm{CH}_{2}, \mathrm{C}-\right.$ 2), $22.8\left(\mathrm{CH}_{3}, \mathrm{C}-19\right), 22.8\left(\mathrm{CH}_{2}, \mathrm{C}-6\right), 21.3\left(\mathrm{CH}_{3}\right.$, acetyl C2), $18.4\left(\mathrm{CH}_{3}, \mathrm{C}-30\right), 18.1\left(\mathrm{CH}_{3}, \mathrm{C}-21\right), 16.9\left(\mathrm{CH}_{3}, \mathrm{C}-29\right)$, $16.0\left(\mathrm{CH}_{3}, \mathrm{C}-18\right), 12.0\left(\mathrm{CH}_{3}, \mathrm{C}-27\right), 9.3\left(\mathrm{CH}_{3}\right.$, propionyl C3"); HRMS (ESI-TOF) $\mathrm{m} / z 591.3659$ [M $+\mathrm{Na}]^{+}$(calcd. for $^{\prime \prime}$ $\mathrm{C}_{35} \mathrm{H}_{52} \mathrm{O}_{6} \mathrm{Na}$, 591.3656).

\section{General procedure for the 15-0-acylation of 2: synthesis of 10-12}

Compound 2 (20-45 $\mu \mathrm{mol})$ was treated with corresponding acyl chloride (excess; $50-70 \mu \mathrm{l})$ in pyridine- $\mathrm{CH}_{2} \mathrm{Cl}_{2}(2: 1)$ at room temperature for $3 \mathrm{~h}$. The mixture was diluted with EtOAc and washed with $\mathrm{H}_{2} \mathrm{O}$, and the aqueous layer was 
extracted with EtOAc. The combined EtOAc solution was concentrated under reduced pressure, and the residue was purified by preparative HPLC $\left(\mathrm{MeCN}-\mathrm{H}_{2} \mathrm{O}\right)$ to furnish the 15- $O$-acyl derivative.

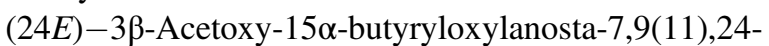
trien-26-oic acid (10): colorless amorphous solid, yield $32 \%$ (3.8 mg); IR (ATR) $\nu_{\max }$ 2929, 1731, 1686, 1375, 1247, $1030 \mathrm{~cm}^{-1}$; ${ }^{1} \mathrm{H}$ NMR $\left(400 \mathrm{MHz}, \mathrm{CDCl}_{3}\right)$, selected resonances, $\delta 6.85(1 \mathrm{H}, \mathrm{t}, J=7.1 \mathrm{~Hz}), 5.47(1 \mathrm{H}, \mathrm{m}), 5.32(1 \mathrm{H}$, $\mathrm{d}, J=5.6 \mathrm{~Hz}), 5.07(1 \mathrm{H}, \mathrm{dd}, J=9.7,4.9 \mathrm{~Hz}), 4.50(1 \mathrm{H}, \mathrm{dd}$, $J=11.2,4.4 \mathrm{~Hz}), 2.32(2 \mathrm{H}, \mathrm{t}, J=7.5 \mathrm{~Hz}), 2.06(3 \mathrm{H}, \mathrm{s})$, $1.83(3 \mathrm{H}, \mathrm{s}), 1.17(1 \mathrm{H}, \mathrm{dd}, J=10.7,5.0 \mathrm{~Hz}), 1.00(3 \mathrm{H}, \mathrm{s})$, $0.99(3 \mathrm{H}, \mathrm{s}), 0.97(3 \mathrm{H}, \mathrm{t}, J=7.5 \mathrm{~Hz}), 0.94(3 \mathrm{H}, \mathrm{s}), 0.91$ $(3 \mathrm{H}, \mathrm{d}, J=6.4 \mathrm{~Hz}), 0.89(3 \mathrm{H}, \mathrm{s}), 0.65(3 \mathrm{H}, \mathrm{s}) ;{ }^{13} \mathrm{C} \mathrm{NMR}$ $\left(100 \mathrm{MHz}, \mathrm{CDCl}_{3}\right) \delta 173.5,170.7,170.2,146.1,144.8$, $140.6,126.7,121.3,116.3,80.9,77.0,51.8,49.4,49.2$, $44.4,38.4,37.8,37.6,37.2,36.9,36.1,35.7,34.9,28.2$, 26.0, 24.4, 23.0, 22.9, 21.1, 18.6, 18.5, 18.3, 16.9, 16.2, 13.7, 12.1; HRMS (ESI-TOF) $m / z$ 605.3810 $[\mathrm{M}+\mathrm{Na}]^{+}$ (calcd. for $\mathrm{C}_{36} \mathrm{H}_{54} \mathrm{O}_{6} \mathrm{Na}, 605.3813$ ).

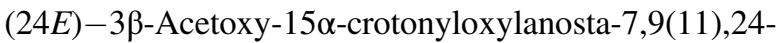
trien-26-oic acid (11): colorless amorphous solid, yield $22 \%$ (5.1 mg); IR (ATR) $\nu_{\max }$ 2940, 1717, 1685, 1375, 1247, 1029, $756 \mathrm{~cm}^{-1}$; ${ }^{1} \mathrm{H} \mathrm{NMR}\left(400 \mathrm{MHz}, \mathrm{CDCl}_{3}\right)$, selected resonances, $\delta 6.99(1 \mathrm{H}, \mathrm{dq}, J=15.6,6.8 \mathrm{~Hz}), 6.84(1 \mathrm{H}, \mathrm{t}$, $J=7.2 \mathrm{~Hz}), 5.90(1 \mathrm{H}, \mathrm{dd}, J=15.6,1.4 \mathrm{~Hz}), 5.43(1 \mathrm{H}, \mathrm{d}, J$ $=5.5 \mathrm{~Hz}), 5.32(1 \mathrm{H}, \mathrm{d}, J=5.8 \mathrm{~Hz}), 5.12(1 \mathrm{H}, \mathrm{dd}, J=10.2$, $5.5 \mathrm{~Hz}), 4.51(1 \mathrm{H}, \mathrm{dd}, J=11.2,4.5 \mathrm{~Hz}), 2.32(1 \mathrm{H}$, br d, $J=$ $17.7 \mathrm{~Hz}), 2.05(3 \mathrm{H}, \mathrm{s}), 1.89(3 \mathrm{H}, \mathrm{d}, J=6.8 \mathrm{~Hz}), 1.82(3 \mathrm{H}$, s), $1.17(1 \mathrm{H}, \mathrm{dd}, J=10.9,4.4 \mathrm{~Hz}), 1.03(3 \mathrm{H}, \mathrm{s}), 0.99(3 \mathrm{H}$, s), $0.94(3 \mathrm{H}, \mathrm{s}), 0.92(3 \mathrm{H}, \mathrm{d}, J=6.4 \mathrm{~Hz}), 0.88(3 \mathrm{H}, \mathrm{s}), 0.66$ $(3 \mathrm{H}, \mathrm{s}) ;{ }^{13} \mathrm{C} \mathrm{NMR}\left(100 \mathrm{MHz}, \mathrm{CDCl}_{3}\right) \delta 172.0,171.0,166.6$, $145.6,145.1,144.5,140.2,126.6,123.2,121.2,116.1$, $80.7,77.2,51.5,48.9,48.9,44.1,38.0,37.6,37.3,35.9$, $36.1,35.3,34.6,28.1,25.9,24.2,22.8,22.8,21.3,18.4$, 18.2, 18.0, 16.9, 16.0, 12.0; HRMS (ESI-TOF) $\mathrm{m} / \mathrm{z}$ $603.3649[\mathrm{M}+\mathrm{Na}]^{+}$(calcd. for $\mathrm{C}_{36} \mathrm{H}_{52} \mathrm{O}_{6} \mathrm{Na}, 603.3656$ ).

(24E)-3 $\beta$-Acetoxy-15 $\alpha$-isobutyryloxylanosta-7,9(11),24trien-26-oic acid (12): colorless amorphous solid, yield $26 \%$ (6.8 mg); IR (ATR) $\nu_{\max }$ 2972, 2932, 1731, 1686, 1375, 1247 $\mathrm{cm}^{-1}$; ${ }^{1} \mathrm{H}$ NMR $\left(400 \mathrm{MHz}, \mathrm{CDCl}_{3}\right.$ ), selected resonances, $\delta$ $6.85(1 \mathrm{H}, \mathrm{t}, J=7.0 \mathrm{~Hz}), 5.46(1 \mathrm{H}, \mathrm{m}), 5.32(1 \mathrm{H}, \mathrm{d}, J=6.0$ $\mathrm{Hz}), 5.05(1 \mathrm{H}, \mathrm{dd}, J=10.0,5.2 \mathrm{~Hz}), 4.51(1 \mathrm{H}, \mathrm{dd}, J=11.3$, $4.5 \mathrm{~Hz}), 2.58(1 \mathrm{H}, \mathrm{m}), 2.32(1 \mathrm{H}, \mathrm{br} \mathrm{d}, J=17.7 \mathrm{~Hz}), 2.06(3 \mathrm{H}$, s), $1.83(3 \mathrm{H}, \mathrm{s}), 1.19(6 \mathrm{H}, \mathrm{d}, J=7.0 \mathrm{~Hz}), 1.01(3 \mathrm{H}, \mathrm{s}), 0.98$ $(3 \mathrm{H}, \mathrm{s}), 0.94(3 \mathrm{H}, \mathrm{s}), 0.92(3 \mathrm{H}, \mathrm{d}, J=6.4 \mathrm{~Hz}), 0.89$ (3H, s), $0.65(3 \mathrm{H}, \mathrm{s}) ;{ }^{13} \mathrm{C} \mathrm{NMR}\left(100 \mathrm{MHz}, \mathrm{CDCl}_{3}\right) \delta 177.1,172.4$, 171.0, 145.6, 145.1, 140.3, 126.7, 121.0, 116.1, 80.7, 77.2, 51.5, 48.9, 48.9, 44.1, 37.9, 37.6, 37.3, 37.1, 36.0, 35.3, 34.6, $34.3,28.1,26.0,24.2,22.8,22.8,21.3,19.0,19.0,18.4,18.2$, 16.9, 16.0, 12.0; HRMS (ESI-TOF) $\mathrm{m} / z$ 605.3814 [M + Na] ${ }^{+}$ (calcd. for $\mathrm{C}_{36} \mathrm{H}_{54} \mathrm{O}_{6} \mathrm{Na}, 605.3813$ ).

\section{Synthesis of 13}

A mixture of $3(5.7 \mathrm{mg}, 11 \mu \mathrm{mol})$ and formic acid $(20 \mu \mathrm{l})$ in 2,2,2-trifluoroethyl formate $(0.2 \mathrm{ml})$ was stirred on a $60{ }^{\circ} \mathrm{C}$ heating bath for $3 \mathrm{~h}$. After cooling to room temperature, the mixture was concentrated in vacuo. The residue was purified by preparative HPLC $\left(\mathrm{MeCN}-\mathrm{H}_{2} \mathrm{O}\right)$ to furnish 13 (3.6 $\mathrm{mg}, 60 \%)$.

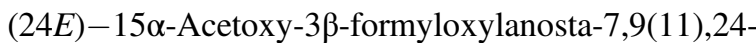
trien-26-oic acid (13): colorless amorphous solid; IR (ATR) $\nu_{\max } 2938,1721,1686,1376,1249,1176 \mathrm{~cm}^{-1} ;{ }^{1} \mathrm{H}$ NMR $\left(400 \mathrm{MHz}, \mathrm{CDCl}_{3}\right)$, selected resonances, $\delta 8.12(1 \mathrm{H}, \mathrm{s})$, $6.84(1 \mathrm{H}, \mathrm{t}, J=7.0 \mathrm{~Hz}), 5.48(1 \mathrm{H}, \mathrm{d}, J=5.6 \mathrm{~Hz}), 5.33(1 \mathrm{H}$, $\mathrm{d}, J=6.0 \mathrm{~Hz}), 5.07(1 \mathrm{H}, \mathrm{dd}, J=10.3,5.3 \mathrm{~Hz}), 4.64(1 \mathrm{H}$, $\mathrm{dd}, J=10.2,5.6 \mathrm{~Hz}), 2.32(1 \mathrm{H}$, br $\mathrm{d}, J=17.6 \mathrm{~Hz}), 2.32$ $(2 \mathrm{H}, \mathrm{t}, J=7.5 \mathrm{~Hz}), 2.09(3 \mathrm{H}, \mathrm{s}), 1.83(3 \mathrm{H}, \mathrm{s}), 1.19(1 \mathrm{H}, \mathrm{dd}$, $J=11.2,4.5 \mathrm{~Hz}), 1.01(3 \mathrm{H}, \mathrm{s}), 1.00(3 \mathrm{H}, \mathrm{s}), 0.97(3 \mathrm{H}, \mathrm{s})$, $0.92(3 \mathrm{H}, \mathrm{s}), 0.91(3 \mathrm{H}, \mathrm{d}, J=6.4 \mathrm{~Hz}), 0.65(3 \mathrm{H}, \mathrm{s}) ;{ }^{13} \mathrm{C}$ NMR $\left(100 \mathrm{MHz}, \mathrm{CDCl}_{3}\right) \delta 171.1,170.2,161.0,145.7$, $144.8,140.4,126.8,121.1,116.3,80.9,77.0,51.4,49.1$, $48.9,44.2,38.1,37.6,37.4,37.0,36.0,35.5,34.7,28.1$, 25.9, 24.4, 22.8, 22.8, 21.3, 18.4, 18.2, 16.9, 16.0, 12.1; HRMS (ESI-TOF) $\mathrm{m} / z \quad 563.3340[\mathrm{M}+\mathrm{Na}]^{+}$(calcd. for $\mathrm{C}_{33} \mathrm{H}_{48} \mathrm{O}_{6} \mathrm{Na}, 563.3343$ ).

\section{General procedure for 3-0-acylation of 3: synthesis of 14-16}

Compound 3 (10-11 $\mu \mathrm{mol})$ was treated with corresponding acyl chloride (excess, $30 \mu \mathrm{l}$ ) in pyridine $(100 \mu \mathrm{l})-\mathrm{CH}_{2} \mathrm{Cl}_{2}$ $(50 \mu \mathrm{l})$ at room temperature for $3 \mathrm{~h}$. The mixture was diluted with EtOAc and washed with $\mathrm{H}_{2} \mathrm{O}$, and the aqueous layer was extracted with EtOAc. The combined EtOAc solution was concentrated under reduced pressure, and the residue was purified by preparative $\mathrm{HPLC}\left(\mathrm{MeCN}-\mathrm{H}_{2} \mathrm{O}\right)$ to furnish the 3-O-acyl derivative.

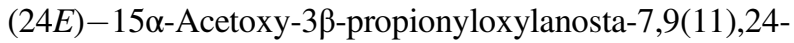
trien-26-oic acid (14): colorless amorphous solid, yield $42 \%$ (2.4 mg); IR (ATR) $\nu_{\max }$ 2923, 1734, 1686, 1376, 1248 $\mathrm{cm}^{-1} ;{ }^{1} \mathrm{H}$ NMR $\left(400 \mathrm{MHz}, \mathrm{CDCl}_{3}\right)$, selected resonances, $\delta$ $6.85(1 \mathrm{H}, \mathrm{t}, J=7.0 \mathrm{~Hz}), 5.47(1 \mathrm{H}, \mathrm{d}, J=5.5 \mathrm{~Hz}), 5.32(1 \mathrm{H}$, $\mathrm{d}, J=6.1 \mathrm{~Hz}), 5.07(1 \mathrm{H}, \mathrm{dd}, J=10.2,5.4 \mathrm{~Hz}), 4.52(1 \mathrm{H}, \mathrm{dd}$, $J=11.2,4.6 \mathrm{~Hz}), 2.33(2 \mathrm{H}, \mathrm{q}, J=7.5 \mathrm{~Hz}), 2.09(3 \mathrm{H}, \mathrm{s})$, $1.83(3 \mathrm{H}, \mathrm{s}), 1.17(1 \mathrm{H}, \mathrm{dd}, J=10.7,4.5 \mathrm{~Hz}), 1.15(3 \mathrm{H}, \mathrm{t}, J$ $=7.5 \mathrm{~Hz}), 1.00(3 \mathrm{H}, \mathrm{s}), 0.98(3 \mathrm{H}, \mathrm{s}), 0.95(3 \mathrm{H}, \mathrm{s}), 0.91(3 \mathrm{H}$, $\mathrm{d}, J=6.4 \mathrm{~Hz}), 0.89(3 \mathrm{H}, \mathrm{s}), 0.65(3 \mathrm{H}, \mathrm{s}) ;{ }^{13} \mathrm{C} \mathrm{NMR}(100$ $\left.\mathrm{MHz} \mathrm{CDCl}_{3}\right) \delta 173.9,171.4,170.8,146.0,144.6,140.5$, $126.5,121.2,116.3,80.5,76.9,51.6,49.3,49.0,44.3,38.2$, $37.8,37.5,37.0,36.0,35.6,34.8,28.1,28.0,25.9,24.3$, 22.9, 22.8, 21.3, 18.3, 18.2, 16.9, 16.1, 12.0, 9.2; HRMS $\left(\right.$ ESI-TOF) $\quad m / z \quad 591.3657 \quad[\mathrm{M}+\mathrm{Na}]^{+} \quad$ (calcd. for $\mathrm{C}_{35} \mathrm{H}_{52} \mathrm{O}_{6} \mathrm{Na}$, 591.3656). 
$(24 E)-15 \alpha$-Acetoxy-3 $\beta$-crotonyloxylanosta-7,9(11),24trien-26-oic acid (15): colorless amorphous solid, yield $22 \%$ $(1.3 \mathrm{mg})$; IR (ATR) $\nu_{\max }$ 2930, 1717, 1686, 1376, 1249, $1181 \mathrm{~cm}^{-1}$; ${ }^{1} \mathrm{H}$ NMR $\left(400 \mathrm{MHz}, \mathrm{CDCl}_{3}\right)$, selected resonances, $\delta 6.96(1 \mathrm{H}, \mathrm{dq}, J=15.5,6.7 \mathrm{~Hz}), 6.84(1 \mathrm{H}, \mathrm{t}, J=$ $7.2 \mathrm{~Hz}), 5.86(1 \mathrm{H}, \mathrm{dd}, J=15.6,1.4 \mathrm{~Hz}), 5.47(1 \mathrm{H}, \mathrm{d}, J=$ $5.9 \mathrm{~Hz}), 5.33(1 \mathrm{H}, \mathrm{d}, J=5.7 \mathrm{~Hz}), 5.06(1 \mathrm{H}, \mathrm{dd}, J=10.3$, $5.4 \mathrm{~Hz}), 4.57(1 \mathrm{H}, \mathrm{dd}, J=11.5,4.2 \mathrm{~Hz}), 2.32(1 \mathrm{H}$, br d, $J=$ $17.7 \mathrm{~Hz}), 2.09(3 \mathrm{H}, \mathrm{s}), 1.88(3 \mathrm{H}, \mathrm{d}, J=6.8 \mathrm{~Hz}), 1.83(3 \mathrm{H}$, s), $1.19(1 \mathrm{H}, \mathrm{dd}, J=11.4,3.9 \mathrm{~Hz}), 1.00(3 \mathrm{H}, \mathrm{s}), 0.99(3 \mathrm{H}$, s), $0.97(3 \mathrm{H}, \mathrm{s}), 0.91(3 \mathrm{H}, \mathrm{d}, J=6.4 \mathrm{~Hz}), 0.90(3 \mathrm{H}, \mathrm{s}), 0.65$ $(3 \mathrm{H}, \mathrm{s}) ;{ }^{13} \mathrm{C} \mathrm{NMR}\left(100 \mathrm{MHz}, \mathrm{CDCl}_{3}\right) \delta 172.0,171.0,166.6$, $145.6,145.1,144.5,140.2,126.6,123.2,121.2,116.1$, $80.7,77.2,51.5,48.9,48.9,44.1,38.0,37.6,37.3,35.9$, 36.1, 35.3, 34.6, 28.1, 25.9, 24.2, 22.8, 22.8, 21.3, 18.4, 18.2, 18.0, 16.9, 16.0, 12.0; HRMS (ESI-TOF) $\mathrm{m} / \mathrm{z}$ $603.3658[\mathrm{M}+\mathrm{Na}]^{+}$(calcd. for $\mathrm{C}_{36} \mathrm{H}_{52} \mathrm{O}_{6} \mathrm{Na}, 603.3656$ ).

(24E) - 15 $\alpha$-Acetoxy-3 $\beta$-isobutyryloxylanosta-7,9

(11),24-trien-26-oic acid (16): colorless amorphous solid, yield 53\% (3.4 mg); IR (ATR) $\nu_{\max } 2935,1731,1686,1376$, $1248 \mathrm{~cm}^{-1}$; ${ }^{1} \mathrm{H} \mathrm{NMR}\left(400 \mathrm{MHz}, \mathrm{CDCl}_{3}\right)$, selected resonances, $\delta 6.86(1 \mathrm{H}, \mathrm{t}, J=7.1 \mathrm{~Hz}), 5.48(1 \mathrm{H}, \mathrm{d}, J=4.8 \mathrm{~Hz})$, $5.33(1 \mathrm{H}, \mathrm{d}, J=5.4 \mathrm{~Hz}), 5.07(1 \mathrm{H}, \mathrm{dd}, J=10.3,5.4 \mathrm{~Hz})$, $4.50(1 \mathrm{H}, \mathrm{dd}, J=11.2,4.8 \mathrm{~Hz}), 2.55(1 \mathrm{H}, \mathrm{m}), 2.32(1 \mathrm{H}, \mathrm{br}$ $\mathrm{d}, J=17.7 \mathrm{~Hz}), 2.09(3 \mathrm{H}, \mathrm{s}), 1.83(3 \mathrm{H}, \mathrm{s}), 1.19(3 \mathrm{H}, \mathrm{d}, J=$ $6.4 \mathrm{~Hz}), 1.17(3 \mathrm{H}, \mathrm{d}, J=6.4 \mathrm{~Hz}), 1.00(3 \mathrm{H}, \mathrm{s}), 0.99(3 \mathrm{H}, \mathrm{s})$, $0.96(3 \mathrm{H}, \mathrm{s}), 0.92(3 \mathrm{H}, \mathrm{d}, J=6.3 \mathrm{~Hz}), 0.89(3 \mathrm{H}, \mathrm{s}), 0.65$ $(3 \mathrm{H}, \mathrm{s}) ;{ }^{13} \mathrm{C} \mathrm{NMR}\left(100 \mathrm{MHz}, \mathrm{CDCl}_{3}\right) \delta 176.6,170.9,170.1$, 146.1, 144.7, 140.6, 126.7, 121.3, 116.3, 80.4, 77.1, 51.7, $49.4,49.1,44.4,38.3,37.9,37.6,37.1,36.1,35.7,34.9$, $34.6,28.2,26.0,24.4,23.0,22.9,21.2,19.1,19.0,18.4$, 18.3, 17.0, 16.1, 12.1; HRMS (ESI-TOF) $m / z$ 605.3814 [M $+\mathrm{Na}^{+}$(calcd. for $\mathrm{C}_{36} \mathrm{H}_{54} \mathrm{O}_{6} \mathrm{Na}, 605.3813$ ).

\section{General procedure for diacylation of 4: synthesis of 17 and 18}

Compound 4 (10-23 $\mu \mathrm{mol})$ was treated with corresponding acyl chloride (excess, $50 \mu \mathrm{l})$ in pyridine $(0.2 \mathrm{ml})-\mathrm{CH}_{2} \mathrm{Cl}_{2}$ $(0.1 \mathrm{ml})$ at room temperature for $3 \mathrm{~h}$. The mixture was diluted with EtOAc and washed with $\mathrm{H}_{2} \mathrm{O}$, and the aqueous layer was extracted with EtOAc. The combined EtOAc solution was concentrated under reduced pressure, and the residue was purified by preparative $\mathrm{HPLC}\left(\mathrm{MeCN}-\mathrm{H}_{2} \mathrm{O}\right)$ to furnish the diacylated derivative.

(24E)-3 $\beta, 15 \alpha$-Dipropionyloxylanosta-7,9(11),24-trien26-oic acid (17): colorless amorphous solid, yield 23\% (3.1 mg); IR (ATR) $\nu_{\max } 2930,1733,1686,1376,1277,1195$ $\mathrm{cm}^{-1} ;{ }^{1} \mathrm{H}$ NMR $\left(400 \mathrm{MHz}, \mathrm{CDCl}_{3}\right.$ ), selected resonances, $\delta$ $6.85(1 \mathrm{H}, \mathrm{t}, J=7.1 \mathrm{~Hz}), 5.46(1 \mathrm{H}, \mathrm{d}, J=4.9 \mathrm{~Hz}), 5.32(1 \mathrm{H}$, $\mathrm{d}, J=6.0 \mathrm{~Hz}), 5.07(1 \mathrm{H}, \mathrm{dd}, J=9.8,5.1 \mathrm{~Hz}), 4.52(1 \mathrm{H}, \mathrm{dd}$, $J=11.2,4.6 \mathrm{~Hz}), 2.36(2 \mathrm{H}, \mathrm{q}, J=7.5 \mathrm{~Hz}), 2.33(2 \mathrm{H}, \mathrm{q}, J=$ $7.5 \mathrm{~Hz}), 2.09(3 \mathrm{H}, \mathrm{s}), 1.83(3 \mathrm{H}, \mathrm{s}), 1.17(3 \mathrm{H}, \mathrm{t}, J=7.5 \mathrm{~Hz})$,
$1.15(3 \mathrm{H}, \mathrm{t}, J=7.5 \mathrm{~Hz}), 1.01(3 \mathrm{H}, \mathrm{s}), 0.99(3 \mathrm{H}, \mathrm{s}), 0.95$ $(3 \mathrm{H}, \mathrm{s}), 0.91(3 \mathrm{H}, \mathrm{d}, J=6.4 \mathrm{~Hz}), 0.89(3 \mathrm{H}, \mathrm{s}), 0.65(3 \mathrm{H}, \mathrm{s})$; ${ }^{13} \mathrm{C}$ NMR $\left(100 \mathrm{MHz}, \mathrm{CDCl}_{3}\right) \delta 174.5,174.3,171.6,145.7$, 145.1, 140.3, 126.6, 121.1, 116.1, 80.4, 77.1, 51.4, 48.9, $48.9,44.1,38.0,37.7,37.3,37.1,36.0,35.4,34.6,28.1$, $28.1,28.1,26.0,24.2,22.8,22.8,18.4,18.2,16.9,16.0$, 12.0, 9.3, 9.3; HRMS (ESI-TOF) $\mathrm{m} / z$ 605.3819 $[\mathrm{M}+\mathrm{Na}]^{+}$ (calcd. for $\mathrm{C}_{36} \mathrm{H}_{54} \mathrm{O}_{6} \mathrm{Na}, 605.3813$ ).

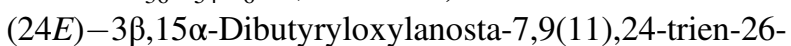
oic acid (18): colorless amorphous solid, yield 48\% (3.0 mg); IR (ATR) $\nu_{\max } 2963,2933,1731,1686,1376,1257$, 1179, $985 \mathrm{~cm}^{-1}$; ${ }^{1} \mathrm{H} \mathrm{NMR}\left(400 \mathrm{MHz}, \mathrm{CDCl}_{3}\right)$, selected resonances, $\delta 6.86(1 \mathrm{H}, \mathrm{t}, J=7.1 \mathrm{~Hz}), 5.47(1 \mathrm{H}, \mathrm{d}, J=4.3$ $\mathrm{Hz}), 5.32(1 \mathrm{H}, \mathrm{d}, J=5.4 \mathrm{~Hz}), 5.07(1 \mathrm{H}, \mathrm{dd}, J=9.8,4.8$ $\mathrm{Hz}), 4.52(1 \mathrm{H}, \mathrm{dd}, J=11.4,4.0 \mathrm{~Hz}), 2.32(2 \mathrm{H}, \mathrm{t}, J=7.2$ $\mathrm{Hz}), 2.29(2 \mathrm{H}, \mathrm{t}, J=7.5 \mathrm{~Hz}), 1.83(3 \mathrm{H}, \mathrm{s}), 1.18(1 \mathrm{H}, \mathrm{dd}$, $J=11.0,4.3 \mathrm{~Hz}), 1.00(3 \mathrm{H}, \mathrm{s}), 0.98(3 \mathrm{H}, \mathrm{s}), 0.97(3 \mathrm{H}, \mathrm{t}$, $J=7.5 \mathrm{~Hz}), 0.96(3 \mathrm{H}, \mathrm{t}, J=7.5 \mathrm{~Hz}), 0.95(3 \mathrm{H}, \mathrm{s}), 0.91(3 \mathrm{H}$, $\mathrm{d}, J=6.3 \mathrm{~Hz}), 0.89(3 \mathrm{H}, \mathrm{s}), 0.65(3 \mathrm{H}, \mathrm{s}) ;{ }^{13} \mathrm{C}$ NMR $(100$ $\left.\mathrm{MHz} \mathrm{CDCl}_{3}\right) \delta 173.8,173.5,171.7,145.6,145.1,140.2$, 126.6, 121.7, 116.1, 80.4, 77.0, 51.4, 48.9, 48.9, 44.0, 37.9, $37.6,37.3,37.1,36.7,36,7,35.9,35.3,34.6,28.1,28.1$, 25.9, 24.2, 22.8, 22.8, 18.6, 18.6, 18.4, 18.1, 17.0, 13.7, 13.7, 12.0; HRMS (ESI-TOF) $m / z 633.4124[\mathrm{M}+\mathrm{Na}]^{+}$ (calcd. for $\mathrm{C}_{38} \mathrm{H}_{58} \mathrm{O}_{6} \mathrm{Na}, 633.4126$ ).

\section{Antitubercular activity against $M$. tuberculosis H37Ra and cytotoxicity of the lanostane triterpenoids}

Growth inhibitory activity of the lanostane triterpenoids against M. tuberculosis H37Ra was evaluated using the green fluorescent protein (GFP)-based microplate assay [13]. Cytotoxicity of the lanostane triterpenoids against Vero cells (African green monkey kidney fibroblasts) was also evaluated using the GFPbased microplate assay [14]. Details of the bioassay procedures are described in the Supplementary material.

\section{Antitubercular activities of compound 9 (GA003) against $M$. tuberculosis H37Rv (virulent strain) and XDR strains}

Activities of compound 9 against M. tuberculosis H37Rv (ATCC 27294, virulent strain) and drug-resistant strains, pre-XDR (THX-0001) and XDR strains (THX-0002, THX0003), were evaluated by the Microplate Alamar Blue Assays [15]. Rifampicin (MIC $0.32 \mu \mathrm{g} \mathrm{ml}^{-1}$ ) and isoniazid (MIC $0.08 \mu \mathrm{g} \mathrm{ml}^{-1}$ ) were used as positive controls for the activity against $M$. tuberculosis H37Rv. Drug-resistant strains of M. tuberculosis used in these assays are clinical isolates from patients in Thailand and preserved at the laboratory of Dr. Angkana Chaiprasert, Drug-Resistant Tuberculosis Research Fund, Siriraj Foundation, Siriraj 
Hospital, Bangkok, Thailand. Details of the bioassay procedures are described in the Supplementary material.

Acknowledgements Financial support from the Thailand Research Fund (Grant No. DBG6280008) is gratefully acknowledged.

\section{Compliance with ethical standards}

Conflict of interest The authors declare no competing interests.

Publisher's note Springer Nature remains neutral with regard to jurisdictional claims in published maps and institutional affiliations.

\section{References}

1. World Health Organization. Global Tuberculosis Report 2020. https://www.who.int/teams/global-tuberculosis-programme/tbreports.

2. Paterson RRM. Ganoderma-a therapeutic fungal biofactory. Phytochemistry. 2006;67:1985-2001.

3. Xia Q, Zhang H, Sun X, Zhao H, Wu L, Zhu D, Yang G, Shao Y, Zhang X, Mao X, Zhang L, She G. A comprehensive review of the structure elucidation and biological activity of triterpenoids from Ganoderma spp. Molecules. 2014;19:17478-535.

4. Baby S, Johnson AJ, Govindan B. Secondary metabolites from Ganoderma. Phytochemistry. 2015;114:66-101.

5. Isaka M, Chinthanom P, Sappan M, Supothina S, Vichai V, Danwisetkanjana K, Boonpratuang T, Hyde KD, Choeyklin R. Antitubercular activity of mycelium-associated Ganoderma lanostanoids. J Nat Prod. 2017;80:1361-9.
6. Isaka $\mathrm{M}$, Chinthanom $\mathrm{P}$, Kongthong $\mathrm{S}$, Srichomthong $\mathrm{K}$, Choeyklin R. Lanostane triterpenes from cultures of the Basidiomycete Ganoderma orbiforme BCC 22324. Phytochemistry. 2013;87:133-9.

7. Isaka M, Chinthanom P, Sappan M, Danwisetkanjana K, Boonpratuang T, Choeyklin R. Antitubercular lanostane triterpenes from cultures of the basidiomycete Ganoderma sp. BCC 16642. J Nat Prod. 2016;79:161-9.

8. Shiao MS, Lin LJ, Yeh SF, Chou CS. Two new triterpenes of the fungus Ganoderma lucidum. J Nat Prod. 1987;50:886-90.

9. Lin LJ, Shiao MS, Yeh SF. Triterpenes from Ganoderma lucidum. Phytochemistry. 1988;27:2269-71.

10. Shiao MS, Lin LJ, Yeh SF. Triterpenes in Ganoderma lucidum. Phytochemistry. 1988;27:873-5.

11. Li YB, Liu RM, Zhong JJ. A new ganoderic acid from Ganoderma lucidum mycelia and its stability. Fitoterapia. 2013;84:115-22.

12. Hill DR, Hsiao C-N, Kurukulasuriya R, Wittenberger SJ. 2,2,2Trifluoroethyl formate: a versatile and selective reagent for the formylation of alcohols, amines, and $N$-hydroxylamines. Org Lett. 2002;4:111-3.

13. Changsen $C$, Franzblau SG, Palittapongarnpim P. Improved green fluorescent protein reporter gene-based microplate screening for antituberculosis compounds by utilizing an acetamidase promoter. Antimicrob Agents Chemother. 2003;47:3682-7.

14. Hunt L, Jordan M, De Jesus M, Wurm FM. GFP-expressing mammalian cells for fast, sensitive, noninvasive cell growth assessment in a kinetic mode. Biotechnol Bioeng. 1999;65:201-5.

15. Collins L, Franzblau SG. Microplate Alamar Blue Assay versus BACTEC 460 system for high-throughput screening of compounds against Mycobacterium tuberculosis and Mycobacterium avium. Antimicrob Agents Chemother. 1997;41:1004-9. 